\title{
Cost-effectiveness of a mobile health-supported lifestyle intervention for pregnant women with an elevated body mass index
}

\author{
Elizabeth O'Sullivan \\ Technological University Dublin, Ireland, liz.osullivan@tudublin.ie \\ Salwa Rokicki \\ University College Dublin \\ Maria Kennelly \\ University College Dublin
}

See next page for additional authors

Follow this and additional works at: https://arrow.tudublin.ie/scschbioart

Part of the Community Health and Preventive Medicine Commons, Health Services Administration Commons, Health Services Research Commons, Maternal and Child Health Commons, Public Health and Community Nursing Commons, and the Women's Health Commons

\section{Recommended Citation}

O'Sullivan, E.J., Rokicki, S., Kennelly, M. et al. Cost-effectiveness of a mobile health-supported lifestyle intervention for pregnant women with an elevated body mass index. Int J Obes 44, 999-1010 (2020). DOI: $10.1038 / \mathrm{s} 41366-020-0531-9$

This Article is brought to you for free and open access by the School of Biological Sciences at ARROW@TU Dublin. It has been accepted for inclusion in Articles by an authorized administrator of ARROW@TU Dublin. For more information, please contact arrow.admin@tudublin.ie, aisling.coyne@tudublin.ie,gerard.connolly@tudublin.ie. Funder: National Maternity Hospital; University College Dublin

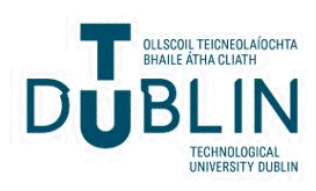


Authors

Elizabeth O'Sullivan, Salwa Rokicki, Maria Kennelly, Kate Ainscough, and Fionnuala M. McAuliffe

This article is available at ARROW@TU Dublin: https://arrow.tudublin.ie/scschbioart/291 


\title{
ARTICLE
}

Health Economics

\section{Cost-effectiveness of a mobile health-supported lifestyle intervention for pregnant women with an elevated body mass index}

\author{
Elizabeth J. O'Sullivan ${ }^{1,2} \cdot$ Slawa Rokicki $^{3,4} \cdot$ Maria Kennelly $^{1} \cdot$ Kate Ainscough $^{1} \cdot$ Fionnuala M. McAuliffe $^{1}$
}

Received: 9 May 2019 / Revised: 5 December 2019 / Accepted: 7 January 2020 / Published online: 21 January 2020

(c) The Author(s), under exclusive licence to Springer Nature Limited 2020

\begin{abstract}
Objective To assess the cost-effectiveness of a mobile health-supported lifestyle intervention compared with usual care. Methods We conducted a cost-effectiveness analysis from the perspective of the publicly-funded health care system. We estimated costs associated with the intervention and health care utilisation from first antenatal care appointment through delivery. We used bootstrap methods to quantify the uncertainty around cost-effectiveness estimates. Health outcomes assessed in this analysis were gestational weight gain (GWG; kg), incidence of excessive GWG, quality-adjusted life years (QALYs), and incidence of large-for-gestational-age (LGA). Incremental cost-effectiveness ratios (ICERs) were calculated as cost per QALY gained, cost per kg of GWG avoided, cost per case of excessive GWG averted, and cost per case of LGA averted.

Results Total mean cost including intervention and health care utilisation was $€ 3745$ in the intervention group and $€ 3471$ in the control group (mean difference $€ 274, P=0.08$ ). The ICER was $€ 2914$ per QALY gained. Assuming a ceiling ratio of $€ 45,000$, the probability that the intervention was cost-effective based on QALYs was 79\%. Cost per kg of GWG avoided was $€ 209$. The cost-effectiveness acceptability curve (CEAC) for kg of GWG avoided reached a confidence level of $95 \%$ at $€ 905$, indicating that if one is willing to pay a maximum of an additional €905 per kg of GWG avoided, there is a $95 \%$ probability that the intervention is cost-effective. Costs per case of excessive GWG averted and case of LGA averted were $€ 2117$ and €5911, respectively. The CEAC for case of excessive GWG averted and for case of LGA averted reached a confidence level of $95 \%$ at $€ 7090$ and $€ 25,737$, respectively.

Conclusions Results suggest that a mobile-health lifestyle intervention could be cost-effective; however, a better understanding of the short- and long-term costs of LGA and excessive GWG is necessary to confirm the results.
\end{abstract}

These authors contributed equally as joint first authors: Elizabeth J. O’Sullivan, Slawa Rokicki

Supplementary information The online version of this article (https:// doi.org/10.1038/s41366-020-0531-9) contains supplementary material, which is available to authorised users.

$\triangle$ Fionnuala M. McAuliffe

fionnuala.mcauliffe@ucd.ie

1 UCD Perinatal Research Centre, School of Medicine, University College Dublin, National Maternity Hospital Ireland, Dublin, Ireland

2 School of Biological and Health Sciences, Technological University Dublin, Dublin, Ireland

3 Geary Institute for Public Policy, University College Dublin, Dublin, Ireland

4 Present address: Department of Health Behavior, Society, and Policy, Rutgers School of Public Health, Piscataway, United States

\section{Introduction}

Poor diet and exercise during pregnancy can result in excessive gestational weight gain (GWG) and the onset of glucose intolerance known as gestational diabetes mellitus (GDM) [1]. Excessive GWG and GDM are associated with costly short- and long-term outcomes for the pregnant woman and her infant. Excessive GWG is associated with increased risk of preterm delivery, as well as large-forgestational-age (LGA) and macrosomic infants [2, 3]. Excessive GWG has also been associated with both an increased risk of early childhood obesity $[4,5]$ and an increase in maternal body mass index (BMI) [6]. This is of concern because obesity is consistently cited as a significant contributor to the global burden of disease and places a significant economic burden on health care systems [7].

Risks for mothers and infants, as well as increased costs to the health care system, have provided the impetus for 
investigators to develop cost-effective interventions to improve diet and exercise and reduce excessive weight gained during pregnancy. This is particularly important among women with an elevated BMI, for whom health service usage and costs during pregnancy are increased by $23 \%$ and $37 \%$, respectively [8], and whose children also incur higher health care costs over their first 18 years of life [9]. A range of lifestyle interventions that promote dietary and physical activity changes have been developed and tested; systematic reviews of the effectiveness of these interventions have concluded that they may be successful at reducing weight gained during pregnancy and risk of preterm birth $[10,11]$; however, little is known regarding how cost-effective these interventions are from the perspective of the health care system.

The objective of this study is to conduct a costeffectiveness analysis to evaluate the costs and consequences of providing a mobile health-supported lifestyle intervention to women with an elevated BMI. To our knowledge, this is the first study to examine the costeffectiveness of using a mobile-phone app to improve maternal and infant outcomes for pregnant women with an elevated BMI.

\section{Methods}

\section{The PEARs trial}

The Pregnancy, Exercise And nutrition Research Study (PEARs) was a single-centre randomised controlled trial conducted within The National Maternity Hospital, Dublin, Ireland; it is registered with Current Controlled Trials (ISRCTN 29316280) and institutional ethical approval was obtained. Participants provided written informed consent. A detailed description of this trial has been published $[12,13]$. Briefly, women $(n=565)$ were recruited at their first antenatal appointment, between 10- and 15-weeks' gestation. Women with a BMI of $\geq 25 \mathrm{~kg} / \mathrm{m}^{2}$ and $\leq 39.9 \mathrm{~kg} / \mathrm{m}^{2}$ were eligible to participate if they were between 18 and 45 years of age, with a singleton pregnancy, and owned a smartphone. Women were excluded if they had experienced GDM in a previous pregnancy, had any illness requiring medical treatment or were unable to provide informed written consent.

\section{Description of the intervention}

The aim of the PEARs trial was to assess whether a 'healthy lifestyle package,' including dietary and exercise advice and a smartphone app to reinforce health messages, would reduce the incidence of GDM. Secondary aims included limiting GWG to within the BMI-specific Institute of
Medicine (IOM) recommendations [14], and reducing the incidence of infants born LGA. Incidence of GDM was assessed at 28 weeks' gestation by an oral glucose tolerance test, total GWG was calculated as the last measured weight between 36 weeks' gestation and delivery minus the weight at the first appointment, and incidence of LGA was defined as birthweight centile $>90$ th centile using GROW centiles [15]. Alongside three visits reinforcing messages about healthy eating and exercise, all women in the intervention group were provided with a smartphone app, where they could find recipes, exercise tips and a 'tip of the day.' The control group received standard care.

\section{Economic evaluation}

The economic evaluation was conducted from the perspective of the publicly-funded health and social care system in Ireland. Analysis was performed by intention-to-treat using all available cases. Costs were calculated from time of first antenatal care appointment through delivery. Unit costs for outpatient visits associated with providing the intervention were calculated based on the amount of time visits took, the health care provider involved, and the average salary of that health care provider, taken from the 2017 Irish Health Service Executive (HSE) salary scales [16]. Total staff costs were adjusted to include social insurance, pension, and overheads, as recommended by the Guidelines for the Economic Evaluation of Health Technologies in Ireland (Table S1) [17]. Number of antenatal admissions and unit costs for medical care associated with antenatal admissions and delivery were derived from Diagnosis-Related Groups (DRGs), recorded by the Hospital In-Patient Enquiry (HIPE) department. After each episode, staff from the HIPE department review patients' charts and input all medical interventions, medications, and visits from health care professionals into their computer system. An algorithm processes this information and assigns each antenatal admission or delivery episode a DRG code, along with the number of days spent in hospital. We obtained these data for each patient in our sample and matched them to the estimated cost per DRG (adjusted for length of stay) that is provided in the HSE Ready Reckoner [18]. Costs provided in the Ready Reckoner are calculated as national averages adjusted to take account of hospital variation. We used these costs to estimate the cost of care for participants in both the intervention and control groups. All hospitals in the Irish health system are currently transitioning to a model of funding that allocates resources to hospitals based on average prices for DRGs (known as "Activity-Based Funding") [19], and is therefore a relevant way of estimating costs in an economic evaluation. Costs of developing the mobile health application were included as in the future the technology would need to be upgraded and re- 
designed to be context-specific; these costs included programming development on both Android and Apple platforms, as well as 30 hours of clinical nutritionist time to develop content. No discounting was applied as the time to follow-up was $<1$ year. Analyses were conducted in $\mathrm{R}$ version 3.5.1 and Stata version 15.1 The code is available upon request.

\section{Outcomes}

A core outcome set for studies on obesity in pregnant patients does not yet exist; a protocol describing a study for its development has recently been published [20]. The health outcomes used in this analysis were GWG $(\mathrm{kg})$, incidence of GWG exceeding IOM guidelines, qualityadjusted life years (QALYs), and incidence of LGA. For binary outcomes, two-sample tests of proportions were used. For continuous outcomes and costs, two-sample $t$ tests were used. Previous analyses of the PEARs trial considered a wide range of primary and secondary outcomes and applied an adjustment for multiple hypothesis testing [13]; in this paper we use a limited set of outcomes, chosen to facilitate comparison with other published studies, and do not adjust for multiple outcomes. We do not explore the cost-effectiveness of the PEARs trial for reducing the incidence of GDM as there was no difference in incidence between the intervention and control groups.

The PEARs trial did not measure QALYs directly. To obtain a measure of QALYs, we converted kilograms of GWG to the expected number of QALYs lost. We applied an analysis from a 2009 IOM report that estimates expected QALYs lost due to outcomes of infant mortality, postpartum weight retention and childhood obesity for each maternal BMI category and value of GWG [21]. Using a range of epidemiological data, the IOM analysis first estimates the probability of each outcome by GWG, controlling for pre-pregnancy BMI category, and the associated expected number of QALYs lost over the lifetime of the mother and child. The analysis then calculates the total expected QALYs lost by GWG and BMI (Table S2 and Fig. S1), which we merged with our data to obtain expected QALYs lost for each participant in the study (rounded to the nearest $\mathrm{kg}$ ). Since the IOM report only calculates QALYs lost for GWG between 0 and $30 \mathrm{~kg}$, we set GWG to $0 \mathrm{~kg}$ for women with negative GWG $(n=5)$ and to $30 \mathrm{~kg}$ for women with GWG $>30 \mathrm{~kg}(n=2)$. We interpreted the difference in QALYs lost between intervention and control groups as the QALYs gained as a result of the intervention.

Incremental cost-effectiveness ratios (ICERs) were calculated as cost per QALY gained, cost per $\mathrm{kg}$ of GWG avoided, cost per case of excessive GWG averted and cost per case of LGA averted. Non-parametric bootstrapping using 5000 resamples was used to characterise uncertainty, which were plotted using cost-effectiveness planes and costeffectiveness acceptability curves (CEAC). Costeffectiveness planes plot the bootstrapped pairs, with the difference in effect on the $x$-axis and the difference in cost on the $y$-axis for the intervention compared with the control. The CEACs represent the probability that the intervention is cost-effective, compared with the control, across a range of willingness-to-pay ratios up to $€ 45,000$ per QALY, which is Ireland's official threshold [22]. The National Institute for Health and Care Excellence (NICE) consider that UK-based interventions that cost the National Health Service less than $£ 20,000$ per QALY gained are cost effective [23].

\section{Sensitivity analysis}

To evaluate the robustness of our findings, we conducted a sensitivity analysis using a per-protocol analysis, in which participants were excluded if they did not attend all three consultation visits. We re-calculated ICERs for all outcomes and compared with our base-case results.

\section{Results}

\section{Sample size}

Five hundred and sixty-five women were recruited into the PEARs trial and were randomly allocated to receive either the 'healthy lifestyle package' or standard care. Nineteen women had no information on delivery costs (ten in control, nine in the intervention group): two had congenital or foetal anomalies, four had miscarriages, eight did not return to the hospital for delivery, and five had missing cost data most likely due to error in assignment of hospital ID numbers. Two hundred and six women (99 in the control and 107 in the intervention groups) were missing gestational weight gain data as a result of not appearing at the antenatal appointment when it was measured. Twenty-four women were missing infant size data (13 in the control and 11 in the intervention). Our analyses included all available cases.

There were no significant differences between the intervention group $(n=278)$ and the control group $(n=287)$ in demographic characteristics at baseline (Table 1). There were also no differences in baseline characteristics between women with recorded gestational weight gain compared to the full sample (Table 1).

\section{PEARs effectiveness}

The proportion of women who developed GDM did not differ between the two groups [13]. Women in the intervention group gained significantly less weight compared with women in the control group $(11.3 \mathrm{~kg}$ vs $12.6 \mathrm{~kg}, P=$ 
Table 1 Baseline characteristics of the PEARs cohort

\begin{tabular}{|c|c|c|c|c|}
\hline \multirow[b]{2}{*}{ Characteristic } & \multicolumn{3}{|l|}{ Full sample } & \multirow{2}{*}{$\begin{array}{l}\text { Participants with GWG data } \\
\text { available } \\
n=359\end{array}$} \\
\hline & $\begin{array}{l}\text { Intervention } \\
(n=278)\end{array}$ & $\begin{array}{l}\text { Control } \\
(n=287)\end{array}$ & All $(n=565)$ & \\
\hline Age-years & $32.8 \pm 4.6$ & $32.2 \pm 4.2$ & $32.5 \pm 4.4$ & $32.4 \pm 4.4$ \\
\hline Primiparous-no. (\%) & $138(49.6)$ & $153(53.3)$ & $291(51.5)$ & $181(50.4)$ \\
\hline \multicolumn{5}{|c|}{ Body mass index category-no. (\%) } \\
\hline Overweight, $25-29.9 \mathrm{~kg} / \mathrm{m}^{2}$ & $184(66.2)$ & $195(67.9)$ & $379(67.1)$ & $243(67.7)$ \\
\hline Obese class I, $30-34.9 \mathrm{~kg} / \mathrm{m}^{2}$ & $71(25.5)$ & $73(25.4)$ & $144(25.5)$ & $90(25.1)$ \\
\hline Obese class II, $\geq 35 \mathrm{~kg} / \mathrm{m}^{2}$ & $23(8.3)$ & $19(6.6)$ & $42(7.4)$ & $26(7.2)$ \\
\hline \multicolumn{5}{|l|}{ Educational attainment—no. (\%) } \\
\hline Some 2nd-level & $7(2.7)$ & $6.0(2.2)$ & $13(2.4)$ & $9(2.6)$ \\
\hline Completed 2nd-level & 35 (13.4) & $35(12.6)$ & $70(13.0)$ & 47 (13.6) \\
\hline Some 3rd-level & $67(25.6)$ & $46(16.6)$ & $113(21.0)$ & $72(20.9)$ \\
\hline Completed 3rd-level & $153(58.4)$ & $190(68.6)$ & $343(63.6)$ & $217(62.9)$ \\
\hline Ethnicity is white-no. (\%) & $257(94.8)$ & $255(91.1)$ & $512(92.3)$ & $324(92.1)$ \\
\hline
\end{tabular}

Age missing for four control and seven intervention participants. Education missing for 10 control and 16 intervention participants. Ethnicity missing or unspecified for seven control and seven intervention participants.

Plus-minus values are means \pm standard deviation, $G W G$ gestational weight gain.
0.03). Women in the intervention group were also less likely to exceed IOM GWG guidelines $(50.9 \%$ vs $63.8 \%$, $P=0.01)$. When we converted absolute GWG to QALYs lost, women in the intervention group lost fewer QALYs, though the difference was not statistically significant $(2.75$ vs $2.85, P=0.38$ ). Finally, infants of mothers in the intervention group were significantly less likely to be born LGA than infants born to mothers in the control group $(4.1 \%$ vs $8.7 \%, P=0.03)$.

\section{Costs}

The cost associated with providing the mobile health intervention was $€ 152$ per person (Table 2 ). There were no significant differences across intervention and control groups in mean cost of antenatal admissions $(P=0.42)$, delivery costs $(P=0.63)$ or total health care utilisation $(P=0.43)$. Total cost of the intervention including health care utilisation was slightly higher in the intervention group, though this difference was not statistically significant $(P=0.08)$.

\section{Cost-effectiveness}

The ICER for QALYs was €2914 per QALY gained (Table 3). More than $77 \%$ of bootstrap pairs were located in the northeast (NE) quadrant, indicating that the PEARs intervention is likely to be more expensive and more effective with regards to QALYs than usual care. At a threshold of $€ 20,000$ per QALY, the probability that PEARs is cost-effective is $77 \%$ (Fig. 1), while at $€ 45,000$ per QALY, the probability is 79\%. The ICER for GWG was $€ 209$ per kg of weight avoided and the ICERs for excessive
GWG and LGA were $€ 2117$ and $€ 5911$ per case averted, respectively (Table 3 ). $95 \%$ or more of bootstrap pairs were located in the NE quadrant for these three outcomes. The CEAC for $\mathrm{kg}$ of GWG avoided reached a confidence level of $95 \%$ at $€ 905$, indicating that if one is willing to pay a maximum of an additional $€ 905$ per $\mathrm{kg}$ of GWG avoided, there is a $95 \%$ probability that the intervention is costeffective (Fig. 2). The CEAC for case of LGA averted reached a confidence level of $95 \%$ at $€ 25,737$ (Fig. 3). The CEAC for case of excessive GWG averted reached a confidence level of $95 \%$ at $€ 7090$.

\section{Sensitivity analysis}

Seventy-two participants were excluded in the per-protocol analysis (32 in the control group, 40 in the intervention group). Results of average costs of health care utilisation in the per-protocol analysis were similar to the base-case analysis (Table S3). Cost of antenatal care and delivery was similar in the intervention and control groups (Table S3). Results on cost-effectiveness were very similar to the basecase analysis, albeit with slightly lower ICERs (Table 3).

\section{Discussion}

\section{Main findings}

This study contributes to our understanding of the costs and consequences of a lifestyle intervention for prevention of GDM; in particular, we are the first study to examine whether using a mobile phone-based programme can be a cost-effective way of improving maternal and infant 
Table 2 Cost of the PEARs intervention and health care utilisation per person (all costs in 2017 euro, €).

\begin{tabular}{|c|c|c|c|c|c|c|}
\hline \multirow[b]{2}{*}{ Cost item } & \multicolumn{2}{|l|}{ Intervention } & \multicolumn{2}{|l|}{ Control } & \multirow[t]{2}{*}{ Difference (SE) } & \multirow[t]{2}{*}{$P$ value } \\
\hline & Mean (SD) & $n$ & Mean (SD) & $n$ & & \\
\hline \multicolumn{7}{|l|}{ Intervention costs } \\
\hline Mobile health application $^{\mathrm{a}}$ & 71.94 & 278 & - & - & - & N/A \\
\hline Consultation visit $1^{\mathrm{b}}$ & 60.28 & 278 & - & - & - & N/A \\
\hline Consultation visit $2^{\mathrm{b}}$ & 7.54 & 278 & - & - & - & N/A \\
\hline Consultation visit $3^{\mathrm{b}}$ & 9.74 & 278 & - & - & - & N/A \\
\hline Consultation emails ${ }^{c}$ & 1.84 & 278 & - & - & - & N/A \\
\hline Text reminders for visits ${ }^{\mathrm{c}}$ & 0.48 & 278 & - & - & - & N/A \\
\hline Total intervention cost per person & 151.82 & & & & & \\
\hline \multicolumn{7}{|l|}{ Health care utilisation costs } \\
\hline Average antenatal admissions costs & $430(913)$ & 269 & $374(706)$ & 276 & $56(69)$ & 0.42 \\
\hline $\begin{array}{l}\text { Delivery costs } \\
\text { Caesarean }+ \text { CSCC }\end{array}$ & $7198(3950)$ & 17 & $6879(2593)$ & 14 & $319(1230)$ & 0.80 \\
\hline Caesarean -CSCC & $4782(91)$ & 56 & $4765(0)$ & 60 & $17(12)$ & 0.14 \\
\hline Vaginal + OR PR + CSCC & $4029(0)$ & 2 & $4029(0)$ & 2 & 0 & 1 \\
\hline Vaginal + OR PR -CSCC & $3036(0)$ & 4 & $3036(0)$ & 3 & 0 & 1 \\
\hline Vaginal & $2320(50)$ & 190 & $2316(0)$ & 198 & $4(4)$ & 0.31 \\
\hline Average delivery costs & 3164 (1739) & 269 & 3097 (1446) & 276 & 73 (137) & 0.63 \\
\hline $\begin{array}{l}\text { Total health care utilisation cost } \\
\text { per person }\end{array}$ & 3593 (1986) & 269 & $3471(1648)$ & 276 & $122(154)$ & 0.43 \\
\hline $\begin{array}{l}\text { Total cost per person, intervention }+ \\
\text { health care utilisation }\end{array}$ & 3745 (1986) & & $3471(1648)$ & & $274(154)$ & 0.08 \\
\hline
\end{tabular}

CSCC Catastrophic or Severe Complication or Comorbidity, OR PR Operating Room or Procedure.

${ }^{a}$ The total cost of the mobile health application was $€ 20,000$, which included both application and content development. We divided total cost by the number of intervention participants.

${ }^{\mathrm{b}}$ The first consultation was with a nutritionist (hourly cost €30.14) and took $\sim 2 \mathrm{~h}$. The second consultation was also with a nutritionist and took $\sim 15 \mathrm{~min}$. The third consultation was with an obstetrician (hourly cost $€ 38.94$ ) and took $\sim 15 \mathrm{~min}$. Costs associated with these consultations were calculated based on the HSE salary scales for these healthcare professionals, including social insurance, pension, and overheads. Details are in the Table S3.

${ }^{\mathrm{c}} \mathrm{A}$ nutritionist was tasked with sending pre-prepared emails to intervention participants every two weeks $(17 \mathrm{~h}$ total $)$ and with tracking the participants to remind them to attend their research-related visits $(4.42 \mathrm{~h}$ total). The cost per participant for these activities was estimated by dividing the total time by the number of intervention participants.

outcomes among pregnant women with an elevated BMI. Our results indicate that the cost of inpatient and outpatient maternal care and delivery did not significantly differ between the treatment and control groups at a threshold of $P<0.05$, even when the cost of the intervention was included. This is important given the clinical benefits observed in terms of reducing GWG and a reduced risk of LGA. Assuming a ceiling ratio of $€ 45,000$, the probability that the intervention was cost-effective was $79 \%$ based on QALYs, indicating that the intervention may be costeffective. The cost per LGA case averted was €5911, while the cost of avoiding an additional kilogram of GWG was $€ 209$ and the cost per case of excess GWG averted was $€ 2117$. As of yet, no detailed analysis of the health effects or costs saved from reduction in risk of LGA or macrosomia exist, which would provide guidance as to a reasonable willingness-to-pay threshold for this outcome.
The 13\% point reduction in risk of excessive GWG observed in this study is clinically relevant as women who gain weight in excess of the IOM recommendations are at increased risk of negative health outcomes, as are their children [21]. Reducing GWG is an important public health goal as, in Ireland, over half of women with an elevated BMI gain excessive weight during pregnancy [2, 24]. Reducing GWG could provide substantial savings to the health care system by reducing a woman's risk of future weight retention $[25,26]$ and lifetime risk of obesity and type 2 diabetes [6, 27]. In addition, infants born LGA, for which excessive GWG is a risk factor independent of maternal BMI [28], are more likely to be obese themselves throughout the life course $[4,5,29]$, and are more likely to develop both type 1 and type 2 diabetes [30,31], which increases their lifetime medical costs $[32,33]$. Thus, the PEARs intervention has the potential to result in long-term 
Table 3 Cost-effectiveness estimates of PEARs (all costs in euro, €).

\begin{tabular}{|c|c|c|c|c|c|c|c|c|c|}
\hline & \multicolumn{2}{|l|}{ Sample size } & \multicolumn{3}{|l|}{ ICER analysis } & \multicolumn{4}{|c|}{$\begin{array}{l}\text { Distribution on cost- } \\
\text { effectiveness plane }(\%)\end{array}$} \\
\hline & Intervention & Control & Incremental cost & Incremental effect & ICER & $\mathrm{NE}$ & SE & SW & NW \\
\hline \multicolumn{10}{|l|}{ Main analysis } \\
\hline QALYs gained & 171 & 188 & 274 & $0.09(-0.12$ to 0.31$)$ & 2914 & 77.1 & 3.1 & 0.7 & 19.1 \\
\hline GWG (kg reduced) & 171 & 188 & 274 & $1.3(0.15-2.49)$ & 209 & 95.0 & 3.8 & 0.0 & 1.1 \\
\hline Excessive GWG (risk reduced) & 171 & 188 & 274 & $0.13(0.03-0.23)$ & 2117 & 95.7 & 3.8 & 0.0 & 0.5 \\
\hline LGA (risk reduced) & 267 & 274 & 274 & $0.046(0.01-0.09)$ & 5911 & 94.9 & 3.8 & 0.1 & 1.3 \\
\hline \multicolumn{10}{|l|}{ Per protocol analysis } \\
\hline QALYs gained & 154 & 172 & 210 & $0.12(-0.10$ to 0.34$)$ & 1723 & 77.0 & 9.2 & 1.3 & 12.5 \\
\hline GWG (kg reduced) & 154 & 172 & 210 & $1.4(0.18-2.6)$ & 154 & 88.5 & 10.4 & 0.1 & 1.0 \\
\hline Excessive GWG (risk reduced) & 154 & 172 & 210 & $0.15(0.04-0.25)$ & 1440 & 89.0 & 10.5 & 0.0 & 0.5 \\
\hline LGA (risk reduced) & 233 & 248 & 210 & $0.046(0.002-0.09)$ & 4592 & 87.6 & 10.2 & 0.3 & 1.9 \\
\hline
\end{tabular}

$G W G$ gestational weight gain, $L G A$ large-for-gestational-age, $Q A L Y$ Quality adjusted life year, $I C E R$ incremental cost-effectiveness ratio, $N E$ northeast, $S E$ southeast, $S W$ southwest, $N W$ northwest.

savings. However, our analysis is limited to the time period from first antenatal care appointment to delivery and we are not able to measure the reduction in health care costs in the long-term for either mother or infant via reduced GWG. We are therefore not able to determine whether the health care service would see long-term savings as a result of this intervention.

An important consideration of our analysis is the choice of the health care system perspective, rather than a societal perspective. A societal perspective would also take into account participant costs, including travel costs, diet and exercise costs, and opportunity costs. We chose the health care system perspective for several reasons. First, it is specified as the reference case perspective in both the guidelines of the Health Information and Quality Authority in Ireland and the NICE in the UK. This perspective therefore facilitates comparison of cost-effectiveness across economic evaluations of health interventions with different health outcomes. Second, the long-run aim of this study would be to integrate PEARs into routine maternity services. In this case, participants would conduct their PEARs visits during the course of their normal antenatal care, and therefore would not incur extra travel costs or opportunity costs for their time on these visits. The phone application itself is able to be used at any time that is convenient for participants, and therefore minimises opportunity costs for women compared to other lifestyle interventions. It is possible, however, that participants would incur higher diet and exercise costs. While the PEARs study was not designed to measure these costs in detail, we do have some evidence that these costs were not substantially increased for participants. In their exit interview, participants were asked whether they felt that their weekly grocery bill increased as a result of the intervention, with responses on a 5-point
Likert scale from strongly agree to strongly disagree. Most participants responded that their costs did not increase: $77 \%$ of participants agreed or strongly agreed that their diet costs did not increase as a result of the intervention, $11 \%$ did not agree or disagree with the statement, and $11 \%$ believed that their costs did increase. However, it is important to acknowledge this limitation in that patient costs were not considered in this analysis.

While there are many randomised trials evaluating the effectiveness of lifestyle interventions for prevention of GDM, few examine cost-effectiveness [10, 11, 34]. We identified three trials with associated economic evaluations. Similar to our results, none of the trials identified reported significant effects on QALYs. The results reported in this study on cost-effectiveness with respect to infant outcomes were consistent with those observed in the NELLI trial in Finland [35] and the LIMIT study in Australia [36]; both trials focused on dietary and physical activity counselling. However, FitFor2, a trial conducted in the Netherlands [37] that focused solely on physical activity was both costlier and less effective than other trials, suggesting that dietary counselling in lifestyle interventions is an important aspect of cost-effectiveness. Although the PEARs trial involved a similar number of total contacts with the health care system as the NELLI and LIMIT trials, PEARs participants had access to a mobile phone application that allowed them to access information on diet and exercise at any time. This additional intervention component did not result in an intervention that was costlier than others described in the literature, but did result in a larger effect on GWG. Participants in the PEARs trial used the app often: the median (IQR) of total instances of use was $24(8-72)$ and the median (IQR) for instances of use per week was 1.75 (0.71-3.49). Consistent access to information, in 
Fig. 1 Cost-effectiveness plane (top) and acceptability curve (bottom) for quality-adjusted life years (QALYs). The top figure shows the difference in QALYs gained on the $x$-axis and the difference in cost on the $y$ axis for the intervention compared with the control. The bottom figure represents the probability that the intervention is cost-effective, compared with the control, for a given willingness-to-pay ratio.
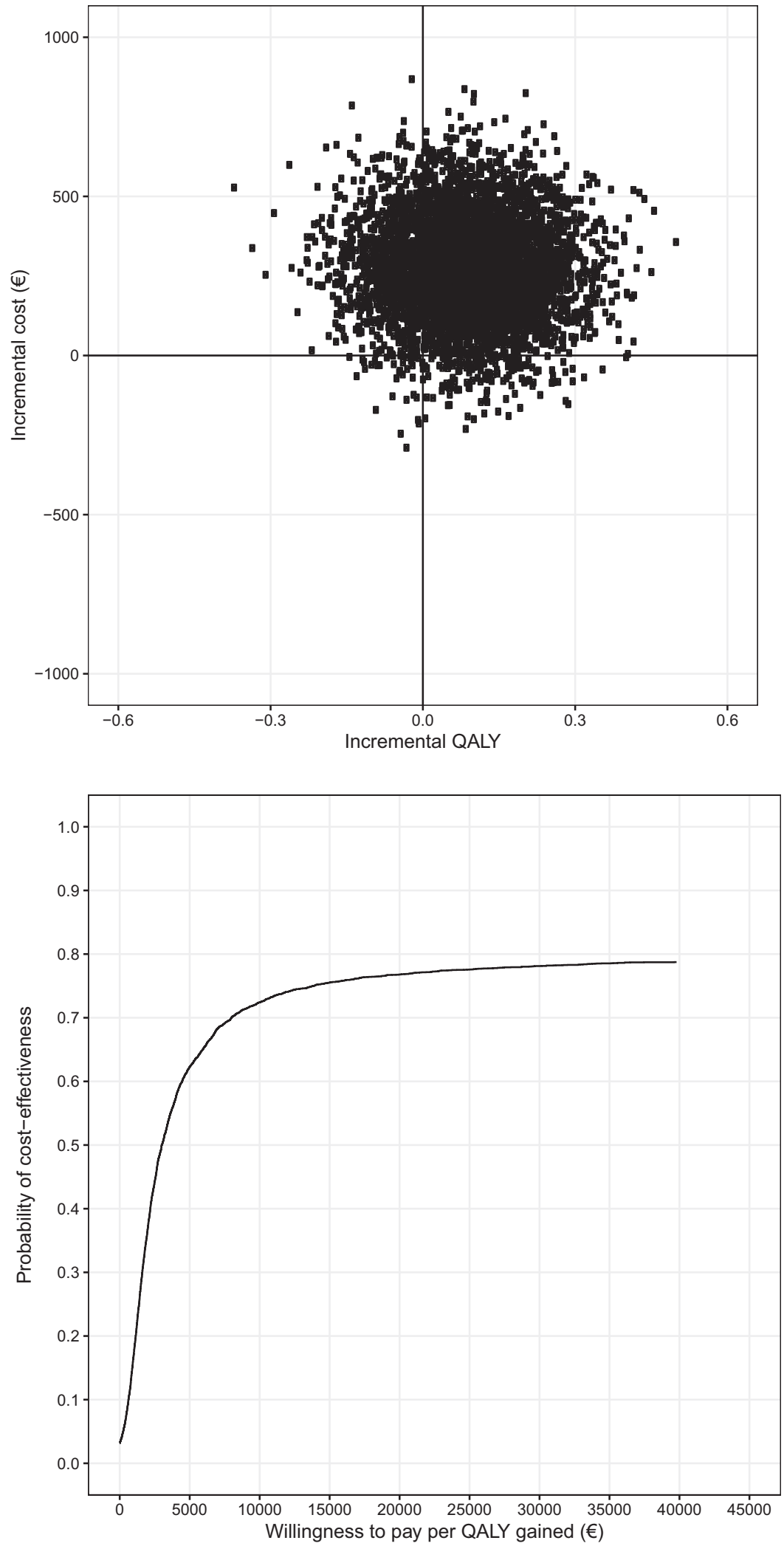

conjunction with regular meetings with health care staff, may have improved GWG outcomes at a low cost to the health care system. A randomised trial of a text messaging intervention for pregnant women in Australia also found significant reductions in GWG [38]. Lastly, a large proportion of the intervention's cost per participant was for 
Fig. 2 Cost-effectiveness plane (top) and acceptability curve (bottom) for gestational weight gain (GWG). The top figure

shows the difference in $\mathrm{kg}$ in weight gain on the $x$-axis and the difference in cost on the $y$-axis for the intervention compared with the control. The bottom figure represents the probability that the intervention is costeffective, compared with the control, for a given willingnessto-pay ratio.
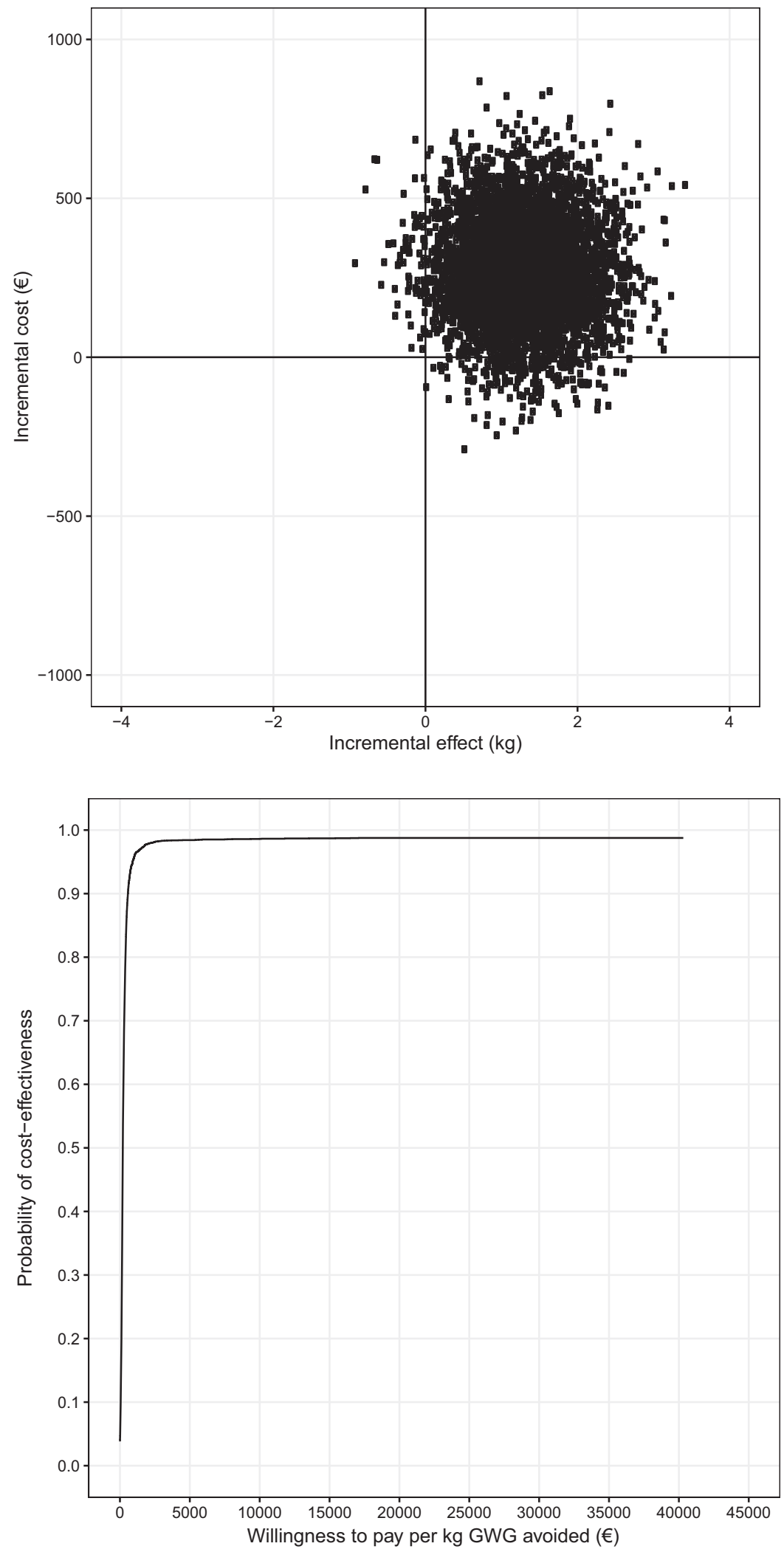

development of the mobile application; the intervention may be more cost-effective when considering scaling up its use to a larger population.
It is possible that it is more effective in terms of health outcomes to conduct an intervention pre-pregnancy with the aim of increasing the proportion of women beginning 
Fig. 3 Cost-effectiveness plane (top) and acceptability curve (bottom) for case of large-forgestational age (LGA). The top figure shows the difference in risk of LGA on the $x$-axis and the difference in cost on the $y$ axis for the intervention compared with the control. The bottom figure represents the probability that the intervention is cost-effective, compared with the control, for a given willingness-to-pay ratio.
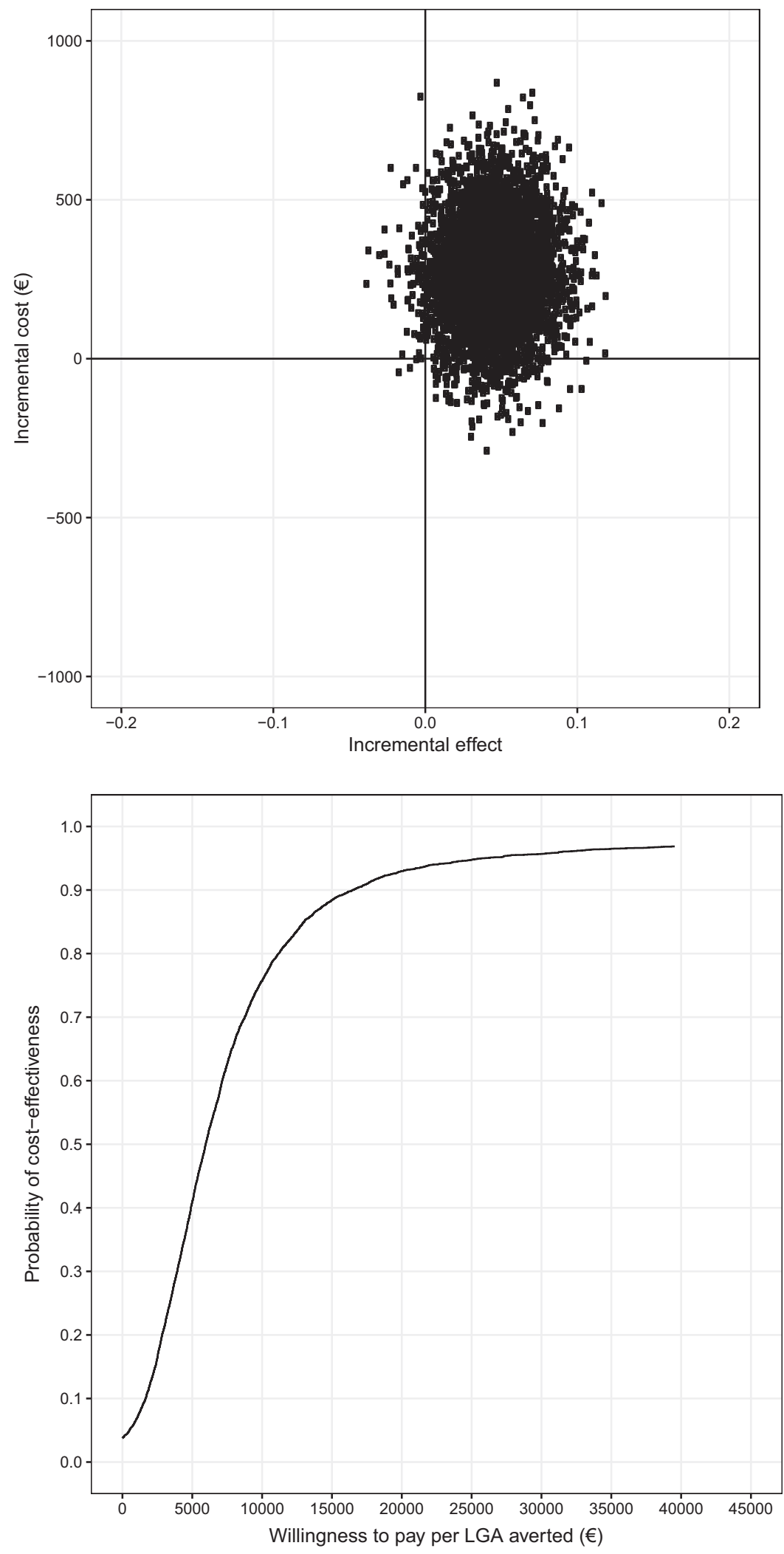

pregnancy with a BMI within the normal range. This is likely to be most beneficial in terms of QALYs; previous research suggests that among women with a normal BMI, increasing GWG does not lead to an increase in QALYs lost to the same extent it does among women with an elevated BMI [21]. However, identifying women planning a 
pregnancy and following them up over time would require considerable time and such an intervention would likely be very costly. Women typically do not access preconception health services [39]; as such, a sustained period of increased contact points with health care professionals-i.e. during pregnancy-is still likely to be the most effective time to intervene. An alternative to conducting an intervention prepregnancy would be to conduct an intervention among women with a BMI within the normal range with an aim of limiting their GWG, which would reduce their risk of beginning future pregnancies at an elevated BMI. A protocol for such a trial has been published [40].

\section{Strengths and limitations}

This analysis makes an important contribution to the literature as there is currently a dearth of cost-effectiveness information available for lifestyle interventions to reduce risk of GDM. If interventions are to be scaled-up and implemented within health care systems, an understanding of the costs and benefits is essential.

This study has limitations. First, costs were calculated only from pregnancy to delivery; downstream medical costs could substantially impact cost-effectiveness estimates. A seven-year follow up of the NELLI trial found that the mean cumulative cost of the intervention group was $30.6 \%$ lower than in the usual care group; however, this difference was not statistically significant [41]. Second, we considered the health care system perspective in our evaluation to provide a policy-relevant analysis. A societal perspective that includes patient costs may impact on the cost-effectiveness results.

Third, we do not measure QALYs directly, but estimate QALYs from secondary data. Modelling QALYs lost using secondary data has several limitations. First, it introduces uncertainty into our analysis because the link between GWG and QALYs was estimated with available IOM data, the data in that analysis were gathered from a variety of sources with varying sample size, and the respondents in the IOM data may not be representative of the participants in PEARs. Second, the IOM analysis focused on several key endpoints (infant mortality, postpartum weight retention, and childhood obesity) because these were deemed to be quantitatively important and reasonably estimated [21]. However, other aspects of quality of life such as mental health, social functioning, and pain would not be included in this analysis. While measuring QALYs directly would be preferable, our results are similar to those of three other lifestyle intervention trials that used three different methods for measuring QALYS — via the health-related quality of life (HRQoL) 15D questionnaire [35], via the SF36 health survey [36], and via the EuroQol-5D questionnaire [37]. None found a significant impact on QALYs and our point estimate lies in between the estimates calculated in these studies.
Finally, the results of this study may not be generalisable to other populations, where health care costs and phone ownership usage may be different.

\section{Conclusion}

Providing a mobile health-supported lifestyle intervention to pregnant women with an elevated BMI may be a costeffective way of improving maternal and infant health. Formative qualitative research exploring the factors influencing dietary choices among pregnant women with an elevated BMI will help inform future intervention development [42]. Additional research on the cost-effectiveness of lifestyle interventions is needed to understand the mechanisms of costeffective interventions and the long-term costs and consequences for both mother and child. It may be prudent to conduct interventions aiming to increase the proportion of women beginning pregnancy with a BMI within the normal range. Such interventions may be more beneficial for both maternal and infant health and more cost-effective.

Funding The PEARs trial received funding from The National Maternity Hospital Medical Fund (Dublin, Ireland) and University College Dublin (Ireland), the authors received no additional funding to conduct the present analysis.

Author contributions EJOS and SR were involved in the conception, planning, carrying out, interpreting and writing up of the work. SR conducted cost-effectiveness analyses. MK and KA were involved in the conception and writing up of the work. FMcA oversaw all aspects of the work and is responsible for the final content.

\section{Compliance with ethical standards}

Conflict of interest The authors declare that they have no conflict of interest.

Publisher's note Springer Nature remains neutral with regard to jurisdictional claims in published maps and institutional affiliations.

\section{References}

1. Motee A, Jeewon R. Importance of exclusive breastfeeding and complementary feeding among infants. Curr Res Nutr Food Sci. 2014;2:17.

2. Walsh JM, McGowan CA, Mahony RM, Foley ME, McAuliffe FM. Obstetric and metabolic implications of excessive gestational weight gain in pregnancy. Obesity. 2014;22:1594-1600.

3. Chu SY, Callaghan WM, Bish CL, D'Angelo D. Gestational weight gain by body mass index among US women delivering live births, 2004-2005: fueling future obesity. Am J Obstet Gynecol. 2009;200:271.e271-271.e277.

4. Mamun A, Mannan M. Gestational weight gain in relation to offspring obesity over the life course: a systematic review and bias-adjusted meta-analysis. Obes Rev. 2014;15:338-47.

5. Schack-Nielsen L, Michaelsen KF, Gamborg M, Mortensen EL, Sørensen TI. Gestational weight gain in relation to offspring body 
mass index and obesity from infancy through adulthood. Int $\mathbf{J}$ Obes. 2010;34:67.

6. Amorim AR, Rössner S, Neovius M, Lourenço PM, Linné Y. Does excess pregnancy weight gain constitute a major risk for increasing long-term BMI? Obesity. 2007;15:1278-86.

7. Withrow D, Alter DA. The economic burden of obesity worldwide: a systematic review of the direct costs of obesity. Obes Rev. 2011;12:131-41.

8. Morgan KL, Rahman MA, Hill RA, Khanom A, Lyons RA, Brophy ST. Obesity in pregnancy: infant health service utilisation and costs on the NHS. BMJ Open. 2015;5:1.

9. Kuhle S, Muir A, Woolcott CG, Brown MM, McDonald SD, Abdolell M, et al. Maternal pre-pregnancy obesity and health care utilization and costs in the offspring. Int J Obes. 2019;43:735-43.

10. Bain E, Crane M, Tieu J, Han S, Crowther CA, Middleton P. Diet and exercise interventions for preventing gestational diabetes mellitus. Cochrane Database Syst Rev. 2015;1:CD010443. https:// doi.org/10.1002/14651858.CD010443.pub2.

11. Tieu J, Shepherd E, Middleton P, Crowther CA. Dietary advice interventions in pregnancy for preventing gestational diabetes mellitus. Cochrane Database Syst Rev. 2017;1:1.

12. Kennelly MA, Ainscough K, Lindsay K, Gibney E, Mc Carthy M, McAuliffe FM. Pregnancy, exercise and nutrition research study with smart phone app support (Pears): study protocol of a randomized controlled trial. Contemp Clin Trials. 2016;46:92-9.

13. Kennelly MA, Ainscough K, Lindsay KL, O'Sullivan E, Gibney ER, McCarthy M, et al. Pregnancy exercise and nutrition with smartphone application support: a randomized controlled trial. Obstet Gynecol. 2018;131:818-26.

14. National Research Council. Weight gain during pregnancy: reexamining the guidelines. National Academies Press, Birmingham, UK, 2010.

15. Gardosi J, Francis A. Customised weight centile calculator. GROW version 6.7.8.5(IE). Gestation Network, Birmingham, UK, 2016.

16. Health Service Executive. April 2017 revised consolidated payscales. In Edited by Health Service Executive; 2017.

17. Health Information and Quality Authority. Guidelines for the economic evaluation of health technologies in Ireland. Dublin: HIQA; 2014.

18. National Casemix Pogramme of the Health Services Executive. Ready reckoner of acute hospital inpatient and daycase activity \& costs (summarised by DRG) relating to 2011 costs and activity. In. Edited by Programme NC; 2013.

19. Health Service Executive. Activity-based funding programme: Implementation Plan 2015-2017. Ireland, Health Service Executive, 2015. https://assets.gov.ie/40185/74ffe4e1dc104cd6856b5a 32375d8d5f.pdf. Accessed 15 Jan 2020.

20. Dadouch R, Faheim M, Juando-Prats C, Parsons J, D'Souza R. COSSOPP Investigators Development of a Core Outcome Set for Studies on Obesity in Pregnant Patients (COSSOPP): a study protocol. Trials. 2018;19:655

21. Hammitt JK. Optimal gestational weight gain: risk tradeoff calculations. Appendix G, Part IV, 674-683 in Institute of Medicine Committee on Reexamination of Pregnancy Weight Guidelines, Weight gain during pregnancy: reexamining the guidelines. Washington, D.C.: National Academies Press; 2009.

22. O'Mahony JF, Coughlan D. The Irish cost-effectiveness threshold: does it support rational rationing or might it lead to unintended harm to Ireland's health system? PharmacoEconomics. 2016;34:5-11.

23. National Institute for Health and Care Excellence. Developing NICE guidelines: the manual. London, UK: National Institute for Health and Care Excellence; 2018.
24. Heery E, Kelleher CC, Wall PG, McAuliffe FM. Prediction of gestational weight gain-a biopsychosocial model. Public Health Nutr. 2015;18:1488-98.

25. Haugen M, Brantsæter AL, Winkvist A, Lissner L, Alexander J, Oftedal B, et al. Associations of pre-pregnancy body mass index and gestational weight gain with pregnancy outcome and postpartum weight retention: a prospective observational cohort study. BMC Pregnancy Childbirth. 2014;14:201.

26. Nehring I, Schmoll S, Beyerlein A, Hauner H, von Kries R. Gestational weight gain and long-term postpartum weight retention: a meta-analysis. Am J Clin Nutr. 2011;94:1225-31.

27. Rooney BL, Schauberger CW, Mathiason MA. Impact of perinatal weight change on long-term obesity and obesity-related illnesses. Obstet Gynecol. 2005;106:1349-56.

28. Ferraro Z, Barrowman N, Prud'Homme D, Walker M, Wen S, Rodger $\mathrm{M}$, et al. Excessive gestational weight gain predicts large for gestational age neonates independent of maternal body mass index. J Matern-Fetal Neonatal Med. 2012;25:538-42.

29. Yu Z, Han S, Zhu G, Zhu C, Wang X, Cao X, et al. Birth weight and subsequent risk of obesity: a systematic review and metaanalysis. Obes Rev. 2011;12:525-42.

30. Harder T, Roepke K, Diller N, Stechling Y, Dudenhausen JW, Plagemann A. Birth weight, early weight gain, and subsequent risk of type 1 diabetes: systematic review and meta-analysis. Am J Epidemiol. 2009;169:1428-36.

31. Harder T, Rodekamp E, Schellong K, Dudenhausen JW, Plagemann A. Birth weight and subsequent risk of type 2 diabetes: a meta-analysis. Am J Epidemiol. 2007;165:849-57.

32. Dee A, Kearns K, O’Neill C, Sharp L, Staines A, O'Dwyer V, et al. The direct and indirect costs of both overweight and obesity: a systematic review. BMC Res Notes. 2014;7:242.

33. Zhuo $X$, Zhang $P$, Hoerger TJ. Lifetime direct medical costs of treating type 2 diabetes and diabetic complications. Am J Prev Med. 2013;45:253-61.

34. Han S, Middleton P, Crowther CA. Exercise for pregnant women for preventing gestational diabetes mellitus. Cochrane Database Syst Rev. 2012;1:CD009021. https://doi.org/10.1002/14651858.CD009021.pub2.

35. Kolu P, Raitanen J, Rissanen P, Luoto R. Cost-effectiveness of lifestyle counselling as primary prevention of gestational diabetes mellitus: findings from a cluster-randomised trial. PLoS ONE. 2013;8:1-12.

36. Dodd JM, Ahmed S, Karnon J, Umberger W, Deussen AR, Tran $\mathrm{T}$, et al. The cost-effectiveness of providing antenatal lifestyle advice for women who are overweight or obese: the LIMIT randomised trial. BMC Obes. 2015;2:14.

37. Oostdam N, Bosmans J, Wouters MG, Eekhoff EM, van Mechelen W, van Poppel MN. Cost-effectiveness of an exercise program during pregnancy to prevent gestational diabetes: results of an economic evaluation alongside a randomised controlled trial. BMC Pregnancy Childbirth. 2012;12:64.

38. Willcox J, Wilkinson S, Lappas M, Ball K, Crawford D, McCarthy E, et al. A mobile health intervention promoting healthy gestational weight gain for women entering pregnancy at a high body mass index: the txt 4 two pilot randomised controlled trial. BJOG: Int J Obstet Gynaecol. 2017;124:1718-28.

39. Stephenson J, Patel D, Barrett G, Howden B, Copas A, Ojukwu O, et al. How do women prepare for pregnancy? Preconception experiences of women attending antenatal services and views of health professionals. PLoS ONE. 2014;9:e103085.

40. Dodd JM, Deussen AR, Louise J. Optimising gestational weight gain and improving maternal and infant health outcomes through antenatal dietary, lifestyle and physical activity advice: the OPTIMISE randomised controlled trial protocol. BMJ Open. 2018;8:e19583. 
41. Kolu P, Raitanen J, Puhkala J, Tuominen P, Husu P, Luoto R. Effectiveness and cost-effectiveness of a cluster-randomized prenatal lifestyle counseling trial: a seven-year follow-up. PloS ONE. 2016;11:e0167759.
42. O’Brien OA, Lindsay KL, McCarthy M, McGloin AF, Kennelly $\mathrm{M}$, Scully HA, et al. Influences on the food choices and physical activity behaviours of overweight and obese pregnant women: a qualitative study. Midwifery. 2017;47:28-35. 